\title{
Characterization of Pseudo-Boolean Models by Boolean Models and Its Applications to Intermediate Logics
}

\author{
By \\ Masahiko SATO*
}

For the study of intermediate logics, pseudo-Boolean algebras play a very important role as their models. So an investigation into the algebraic structure of pseudo-Boolean models seems essential. For dealing with these models, we already know two operations on models, i.e., Cartesian product and the pile operation. But these operations are incomplete in the sense that there exist finite models which can not be obtained from the two element model $S_{1}$ by these operations alone. There has been a problem of finding a complete set of operations in this sense. (See Hosoi [4], and Hosoi and Ono [8].)

Our main result (Theorem 3.7) solves this problem. More precisely, in $\S 2$, we shall introduce the notion of the patch operation on models, and in $\S 3$, we shall show that Cartesian product and the patch operation are complete in the sense that any finite model can be obtained from $S_{1}$ by these operations.

Further, we shall study intermediate logics through pseudo-Boolean models. The notion of slice defined axiomatically by Hosoi will be characterized algebraically in $\S 4$. To do this, we shall define the notion of the height of pseudo-Boolean models. We shall prove that this height corresponds to the index of slice to which belongs the logic characterized by the model.

In $\S 5$, we shall apply the main result to obtain an easy method for counting the height of models, and a theorem on the immediate predecessors of certain logics.

Communicated by S. Hitotumatu., November 2, 1972.

* Department of Mathematics, University of Tokyo, Hongo, Tokyo. 
Throughout this paper, we expect some familiarity with [5] and [10], since some notations and definitions are borrowed from them without special mentioning.

The author wishes to express his hearty thanks to Profs. T. Hosoi and $\mathrm{H}$. Ono for their helpful suggestions and kind discussions with the author.

\section{$\S 1$. Preliminaries}

Propositional variables are expressed by the letters $a_{0}, a_{1}, \ldots$ By a logic we mean an intermediate propositional logic. By a model we always mean a pseudo-Boolean algebra (PBA) with at least two elements. (See e.g. [1], [12]). We write 1 (0) for the maximum (minimum, respectively) element of a model, where 1 is the designated. We use four logical connectives $\wedge, \vee, \rightarrow$, and $\neg$. Same symbols are used for the corresponding operations in models. It should be noticed that any model $M$ determines a logic $L(M)$, that is, the set of formulas valid in it, and for any logic $L$ there exist a model $M$ such that $L=L(M)$.

Any model $M$ is a partially ordered set by definition. For any elements $p, q(p \leqq q)$ in a model $M$, we write $[p, q]$ for the set $\{x \mid p \leqq x$ $\leqq q\}$, and call it an interval in $M$. It is important to remark that $[p, q]$ is also a PBA by the natural ordering in it (see [2]).

If the ordering in $M$ is linear, we say $M$ is a linear model. We write $L_{n}$ for the linear model with $n+1$ elements. Since any infinite linear model is characteristic for one and the same logic, we write $L_{\omega}$ for such a model. We put $S_{n}=L\left(L_{n}\right)(1 \leqq n \leqq \omega)$.

The following lemma is well-known.

Lemma 1.1. $S_{1} \supsetneq S_{2} \supsetneq \cdots \supsetneq S_{n} \supsetneq \cdots \supsetneq S_{\omega}$.

Clearly, the set $\left\{S_{n} \mid 1 \leqq n \leqq \omega\right\}$ covers all logics which have a linear model.

The following theorem is due to Dummett [3]. We remark here that this theorem can be proved easily by the decomposition theorem of McKay [9] and by Lemma 1.9 in Hosoi and Ono [7].

Theorem 1.2. A logic $L$ has a linear model iff $Z \in L$, where $Z=\left(a_{0}\right.$ 
$\left.\rightarrow a_{1}\right) \vee\left(a_{1} \rightarrow a_{0}\right)$

The definition of slice by Hosoi is as follows:

Definition 1.3. $\mathscr{S}_{n}=\left\{L \mid L+Z=S_{n}\right\}$.

Now we define the pile operation (see also [5]).

Definition 1.4. Let $M, N$ be two models. We define $K=M \uparrow N$ to be the model such that there is some $d \in K$ satisfying the conditions (i) $K=M^{\prime} \cup N^{\prime}$, where $M^{\prime}=\{p \in K \mid p \geqq d\}$ and $N^{\prime}=\{p \in K \mid p \leqq d\}$, (ii) $M$ is isomorphic with $M^{\prime}$, and (iii) $N$ is isomorphic with $N^{\prime}$. By these isomorphisms we identify $M$ with $M^{\prime}$ and $N$ with $N^{\prime}$. Hence $d=0_{M}=1_{N}$.

\section{§2. Patch Operation}

In this section, we first define the patch operation on partially ordered sets. This operation defines an ordered set $R$ from a triple $(P, Q, f)$, where $P, Q$ are partially ordered sets and $f$ is an isomorphism from a subset of $P$ to a subset of $Q$. Afterwards, we consider the case that $P$ and $Q$ are PBAs.

Now let $A$ and $B$ be two disjoint sets and $f: A^{\prime} \rightarrow B^{\prime}$ be a bijection, where $A^{\prime}$ (or $B^{\prime}$ ) is a subset of $A$ (or $B$ ). Define an equivalence relation $\equiv$ on $A \cup B$ by that $x \equiv y$ iff $x=y$ or $x=f(y)$ or $y=f(x)$. We write $A \diamond_{f} B$ for $A \cup B / \equiv$, and call it the patching of $A$ and $B$ by $f$. By identifying those elements in $A \cup B$ that are equivalent w.r.t. $\equiv$, we shall consider that $A \diamond_{f} B=A \cup B$ and $A^{\prime}=B^{\prime}=A \cap B$.

Now suppose that $A$ and $B$ are partially ordered sets and $f: A^{\prime} \rightarrow B^{\prime}$ is an order isomorphism. Then we can define an order $\leqq$ on $A \diamond_{f} B$ as follows:

As mentioned above, we consider that $A \diamond_{f} B=A \cup B$. We denote the ordering of $A$ (or $B$ ) by $\leqq_{A}$ (or $\leqq_{B}$ ). Define a relation $\leqq$ on $A \cup B$ by that $x \leqq y$ iff $\left(x, y \in A\right.$ and $\left.x \leqq_{A} y\right)$ or $\left(x, y \in B\right.$ and $\left.x \leqq_{B} y\right)$ or $(x \in B$, $y \in A$ and for some $z \in A \cap B, x \leqq_{B} z$ and $z \leqq_{A} y$ ) or $(x \in A, y \in B$ and for some $z \in A \cap B, x \leqq_{A} z$ and $z \leqq{ }_{B} y$ ). Then it is easy to see that $\leqq$ is the weakest order on $A \cup B$ which preserves both $\leqq_{A}$ and $\leqq_{B}$. 
Example 2.1. We show an example by Hasse diagrams, where $f$ is an isomorphism which maps $a, b, c$, and $d$ in $A$ to $a, b, c$, and $d$ in $B$.

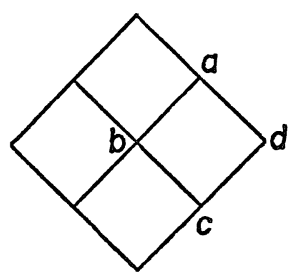

A

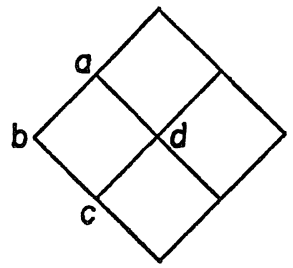

$B$

Fig. 1

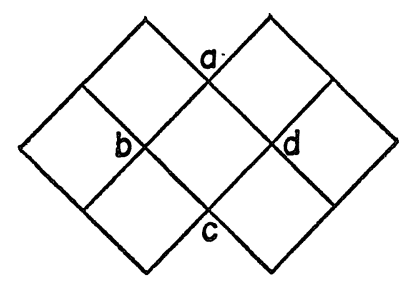

$A \diamond_{f} B$

As seen in the above example, even if $A$ and $B$ are PBAs the patched partially ordered set $A \diamond_{f} B$ is not necessarily a PBA. So, to make $A \diamond_{f} B$ a PBA we must put some restrictions on $f$. The following theorem gives a sufficient condition for $A \diamond_{f} B$ to be a PBA.

Theorem 2.2. If $A$ and $B$ are $P B$ As and $f$ is an isomorphism from an ideal $A^{\prime}$ of $A$ to a filter $B^{\prime}$ of $B$, then $A \diamond_{f} B$ is also a PBA.

Proof. First remark that $A \cap B=\left[0_{A}, 1_{B}\right]$, by the identification stated above. For any $a \in A$ we define $a^{-} \in A \cap B$ by putting $a^{-}=a \wedge_{A} 1_{B}$, and for any $b \in B, b^{+} \in A \cap B$ is defined by $b^{+}=b \vee_{B} 0_{A}$. It is clear that $a^{-} \leqq a$ and $b \leqq b^{+}$. Further, $a \mapsto a^{-}\left(b \mapsto \rightarrow b^{+}\right)$is a homomorphism from $A$ ( $B$, resp.) to $A \cap B$.

(I) Existence of $\inf \{a, b\}$.

Since other cases are trivial, we only consider the case that $a \in A-B$ and $b \in B$. We prove that $\inf \{a, b\}=a^{-} \wedge_{B} b$. Clearly, $a^{-} \wedge_{B} b \leqq b$, and $a^{-} \wedge_{B} b \leqq a^{-} \leqq a$. Hence $a^{-} \wedge_{B} b$ is a lower bound of $\{a, b\}$. On the other hand, let $c$ be any element such that $c \leqq a$ and $c \leqq b$. By the definition of the ordering on $A \diamond_{f} B, c \leqq a$ implies the existence of some $x \in A \cap B$ such that $c \leqq x \leqq a$. Hence $x=x^{-} \leqq a^{-}$. So $c \leqq a^{-}$. Thus $c \leqq a^{-} \wedge_{B} b$. Therefore we see that $a^{-} \wedge_{B} b=\inf \{a, b\}$.

(II) Existence of $\sup \{a, b\}$.

This can be proved as the dual case of (I).

Thus we see that $A \diamond_{f} B$ is a lattice. We denote the lattice operations on $A \diamond_{f} B$ by $\wedge$ and $\vee$. 
(III) Existence of $\max \{x \mid a \wedge x \leqq b\}$.

We consider four cases so that they cover all possible cases. In any of these cases we prove that $\max \{x \mid a \wedge x \leqq b\}=c$ for a certain $c$. We do this in two steps. In (Step 1) we prove that if $a \wedge x \leqq b$ then $x \leqq c$. In (Step 2) we prove that $a \wedge c \leqq b$. From these two steps we have that $\max \{x \mid a \wedge x \leqq b\}=c$. In the following $x$ will denote an arbitrary element such that $a \wedge x \leqq b$.

(i) The case that $a \in A$ and $b \in A$.

We shall prove that $\max \{x \mid a \wedge x \leqq b\}=a \rightarrow{ }_{A} b$.

(Step 1) If $x \in A$ then by the definition of $a \rightarrow{ }_{A} b, x \leqq a \rightarrow{ }_{A} b$. Suppose that $x \in B-A$. Then there is some $y \in A \cap B$ such that $a \wedge x \leqq y \leqq$ b. Hence $a^{-} \wedge_{B} x=a^{-} \wedge x \leqq a \wedge x \leqq y$. So $x \leqq a^{-} \rightarrow_{B} y$. Then since $a^{-} \rightarrow_{B}$ $y \in A \cap B, a \wedge\left(a^{-} \rightarrow_{B} y\right)=a^{-} \wedge\left(a^{-} \rightarrow_{B} y\right) \leqq y$. Hence $a^{-} \rightarrow_{B} y \leqq a \rightarrow_{A} y \leqq a \rightarrow$ ${ }_{A} b$. Thus $x \leqq a \rightarrow{ }_{A} b$.

(Step 2) Obvious.

(ii) The case that $a \in A-B$ and $b \in B-A$.

We shall prove that $\max \{x \mid a \wedge x \leqq b\}=a^{-} \rightarrow{ }_{B} b$.

(Step 1) Suppose that $x \in A$, then $a \wedge x \in A$. Then we have $b \in A$, since $a \wedge x \leqq b \in A$. This contradicts to $b \in B-A$. So we see that $x \in B$. Now, since $a^{-} \leqq a$, we have that $a^{-} \wedge_{B} x=a^{-} \wedge x \leqq a \wedge x \leqq b$. Hence $x \leqq$ $a^{-} \rightarrow{ }_{B} b$.

(Step 2) By definition, $a^{-} \wedge\left(a^{-} \rightarrow{ }_{B} b\right) \leqq b . \quad$ Clearly, $a^{-} \rightarrow_{B} b \in B . \quad$ Then by (I), $a \wedge\left(a^{-} \rightarrow{ }_{B} b\right)=a^{-} \wedge\left(a^{-} \rightarrow{ }_{B} b\right) \leqq b$.

(iii) The case that $a \in B-A$ and $b \in A-B$.

We shall prove that $\max \{x \mid a \wedge x \leqq b\}=a^{+} \rightarrow{ }_{A} b$.

(Step 1) Since $a \wedge x \in B-A$, there is some $y \in A \cap B$ such that $a \wedge$ $x \leqq y \leqq b$. Suppose $x \in B$. Since $a^{+} \wedge\left(a \rightarrow_{B} y\right)=\left(a \wedge\left(a \rightarrow_{B} y\right)\right)^{+} \leqq y^{+}=y$, we have $a \rightarrow_{B} y \leqq a^{+} \rightarrow_{A} y$. Then, $x \leqq a \rightarrow_{B} y \leqq a^{+} \rightarrow_{B} y \leqq a^{+} \rightarrow_{A} y \leqq a^{+} \rightarrow_{A} b$. Next, suppose $x \in A-B$. Then, $a \wedge x^{-}=a \wedge x \leqq b$. Hence $\left(a \wedge x^{-}\right) \vee 0_{A} \leqq b$. Since $a, x^{-}$, and $0_{A}$ are in $B$, we can use the distributive law. Hence, $\left(a \vee 0_{A}\right) \wedge\left(x^{-} \vee 0_{A}\right) \leqq b$. So $a^{+} \wedge x^{-} \leqq b$. Since $a^{+} \in B$ and $x \in A-B, a^{+} \wedge$ $x=a^{+} \wedge x^{-} \leqq b$. Thus we have $x \leqq a^{+} \rightarrow{ }_{A} b$, since $a^{+}, x$, and $b$ are in $A$.

(Step 2) $a \wedge\left(a^{+} \rightarrow{ }_{A} b\right) \leqq a^{+} \wedge\left(a^{+} \rightarrow{ }_{A} b\right) \leqq b$.

(iv) The case that $a \in B$ and $b \in B$.

We shall prove that 


$$
\max \{x \mid a \wedge x \leqq b\}= \begin{cases}1_{B} \rightarrow{ }_{A}\left(a \rightarrow{ }_{B} b\right) & \left(\text { if } a \rightarrow{ }_{B} b \in A\right) \\ a \rightarrow{ }_{B} b & \text { (otherwise) }\end{cases}
$$

First we consider the case that $a \rightarrow_{B} b \in A$. Put $c=1_{B} \rightarrow_{A}\left(a \rightarrow_{B} b\right)$.

(Step 1) If $x \in B$ then $x \leqq a \rightarrow_{B} b \leqq c$. Suppose $x \in A-B$. Then $a \wedge$ $x=a \wedge\left(1_{B} \wedge x\right) \leqq b$. Since $1_{B} \wedge x \in B, 1_{B} \wedge x \leqq a \rightarrow_{B} b$. Since $1_{B}, x$, and $a \rightarrow{ }_{B} b$ are in $A$, we have $x \leqq c$.

(Step 2) By the definition of $c, a \wedge c=a \wedge\left(1_{B} \wedge c\right) \leqq a \wedge\left(a \rightarrow{ }_{B} b\right) \leqq b$.

Next, suppose $c=a \rightarrow{ }_{B} b \in B-A$.

(Step 1) If $x \in B$, then by the definition of $c, x \leqq c$. If $x \in A-B$, then $a \wedge x^{-} \leqq b$, where $x^{-} \in B$. Hence $x^{-} \leqq c \notin A$. Therefore $x^{-} \notin A$. This is a contradiction.

(Step 2) Obvious.

Q.E.D.

From the above proof we have the following table for the caluculation of the logical operators.

\begin{tabular}{c|cll}
$x \wedge y$ & $y \in A-B$ & $y \in A \cap B$ & $y \in B-A$ \\
\hline$x \in A-B$ & $x \wedge_{A} y$ & $x \wedge_{A} y$ & $x \wedge_{B} y$ \\
$x \in A \cap B$ & $x \wedge_{A} y$ & $x \wedge_{A} y$ & $x \wedge_{B} y$ \\
$x \in B-A$ & $x \wedge_{B} y^{-}$ & $x \wedge_{B} y$ & $x \wedge_{B} y$
\end{tabular}

\begin{tabular}{c|cll}
$x \vee y$ & $y \in A-B$ & $y \in A \cap B$ & $y \in B-A$ \\
\hline$x \in A-B$ & $x \vee_{A} y$ & $x \vee_{A} y$ & $x \vee_{A} y^{+}$ \\
$x \in A \cap B$ & $x \vee_{A} y$ & $x \vee_{A} y$ & $x \vee_{B} y$ \\
$x \in B-A$ & $x^{+} \vee_{A} y$ & $x \vee_{B} y$ & $x \vee_{B} y$
\end{tabular}

\begin{tabular}{|c|c|c|c|}
\hline$x \rightarrow y$ & $y \in A-B$ & $y \in A \cap B$ & $y \in B-A$ \\
\hline$x \in A-B$ & $x \rightarrow_{A} y$ & $x \rightarrow_{A} y$ & $x^{-} \rightarrow_{B} y$ \\
\hline$x \in A \cap B$ & $x \rightarrow_{A} y$ & $x \rightarrow_{A} y$ & $x \rightarrow_{B} y$ \\
\hline$x \in B-A$ & $x^{+} \rightarrow_{A} y$ & $1_{B} \rightarrow_{A}\left(x \rightarrow_{B} y\right)$ & $\left\{\begin{array}{l}1_{B} \rightarrow_{A}\left(x \rightarrow_{B} y\right) \\
x \rightarrow_{B} y\end{array}\right.$ \\
\hline
\end{tabular}




\begin{tabular}{c|cc}
$x$ & $\neg x$ \\
\hline$x \in A-B$ & $\left\ulcorner_{B} x^{-}\right.$ & \\
$x \in A \cap B$ & $\neg_{B} x$ & \\
$x \in B-A$ & $1_{B} \rightarrow_{A}\left(\neg_{B} x\right)$ & $\left(\right.$ if $\left.\neg_{B} x \in A\right)$ \\
& $\neg_{B} x$ & $\left(\right.$ if $\left.\neg_{B} x \notin A\right)$
\end{tabular}

Remark. If $f$ is a mapping which identifies $0_{A}$ and $1_{B}$, then $A \diamond_{f} B$ $=A \uparrow B$. Hence the patch operation is a generalization of the pile opera tion.

\section{§3. Completeness of the Patch Operation}

Definition 3.1. A partially ordered set $C$ is called an $n$-cube if it is isomorphic with the $n$-dimensional Boolean algebra.

The following theorem clarifies the local structure of a PBA. This theorem will be used in the last section.

Theorem 3.2. Let be that $P$ is a $P B A$ and $p_{1}, p_{2}, \ldots, p_{n}$ are distinct maximal elements in $P-\{1\}$. Then $\left[p_{1} \wedge \cdots \wedge p_{n}, 1\right]$ is an $n$-cube.

Proof. As an inductive hypothesis, we assume that the theorem holds for $m<n$. Consider an $n$-dimensional Boolean algebra $B=\mathfrak{P}(\{1,2, \ldots, n\})$. We define $\alpha: B \rightarrow\left[p_{1} \wedge \cdots \wedge p_{n}, 1\right]$ by putting $\alpha(K)=\wedge_{i \in K} p_{i}$ for any $K \subset$ $\{1,2, \ldots, n\}$. (We consider $\alpha(\phi)=1$ ) Then, clearly, $\alpha(K \cup L)=\alpha(K) \wedge$ $\alpha(L)$.

We first show that $\alpha$ is injective. To derive a contradiction, let us assume that $K \neq L$ and $\alpha(K)=\alpha(L)$. Then $\alpha(K \cup L)=\alpha(K)$. Hence, without any loss of generality, we have only to consider the case that $K=\{1,2, \ldots, i\}$ and $L=\{1,2, \ldots, i, i+1\}$. That is,

$$
\begin{aligned}
& \alpha(K)=p_{1} \wedge \cdots \wedge p_{i}=p, \\
& \alpha(L)=p \wedge p_{i+1}=p .
\end{aligned}
$$

Hence $p_{i+1} \geqq p_{1} \wedge \cdots \wedge p_{i}$. Now by the inductive hypothesis, $\left[p_{1} \wedge \cdots \wedge p_{i}\right.$, 
$1]$ is an $i$-cube. So, $p_{i+1}$ must coincide with one of $p_{1}, \ldots, p_{i}$, since $p_{i+1}$ is maximal in $\left[p_{1} \wedge \cdots \wedge p_{i}, 1\right]-\{1\}$. This is a contradiction.

Next, we prove that $\alpha$ is surjective. Take any $r \geqq p_{1} \wedge \cdots \wedge p_{n}$. We put $p=p_{1} \wedge \cdots \wedge p_{n}$ and $q_{i}=p_{1} \wedge \cdots \wedge p_{i-1} \wedge p_{i+1} \wedge \cdots \wedge p_{n}$. Then,

$$
r \wedge q_{i} \geqq p \quad(1 \leqq i \leqq n)
$$

First suppose that $r \wedge q_{i}>p$ for some $i$. If $r \geqq q_{i}$ we can prove that there is some $K \subset\left\{p_{1}, \ldots, p_{i-1}, p_{i+1}, \ldots, p_{n}\right\}$ such that $r=\alpha(K)$ by the inductive hypothesis. Hence we may assume $r \geqq q_{i}$. Then we have $q_{i}>r \wedge q_{i}>p$. Since $p_{i} \vee q_{i}=1, p_{i} \wedge q_{i}=p$, and $P$ is a modular lattice we get $1>\left(r \wedge q_{i}\right)$ $\vee p_{i}>p_{i}$. This contradicts that $p_{i}$ is maximal in $P-\{1\}$. Thus, there only remains the case that $r \wedge q_{i}=p$ for any $i$. Then $p=\bigvee_{i=1}^{n}\left(r \wedge q_{i}\right)=r \wedge$ $\bigvee_{i=1}^{n} q_{i}=r \wedge 1=r$. Hence $r=\alpha(\{1,2, \ldots, n\})$.

Thus we have seen that $\alpha: B \rightarrow[p, 1]$ is a bijection. Further, if $K \subset L$, then $\alpha(L)=\alpha(K \cup L)=\alpha(K) \wedge \alpha(L)$. Hence $\alpha(K) \geqq \alpha(L)$. This means that $\alpha$ is an anti-isomorphism. Since $B$ is self-dual, we see that $[p, 1]$ is an $n$-cube.

Q.E.D.

Dually we have the following

Corollary 3.3. Let be that $P$ is a $P B A$ and $p_{1}, p_{2}, \ldots, p_{n}$ are distinct minimal elements in $P-\{0\}$. Then $\left[0, p_{1} \vee \cdots \vee p_{n}\right]$ is an $n$-cube.

For any $p$ in $P$, since $[0, p]$ and $[p, 1]$ can be regarded as PBAs, we can use the above results to investigate the "neighborhood" of $p$.

The operation of patching naturally suggests the "inverse" operation, namely, the cut operation. But, instead of defining the cut operation, we define the notion of section.

Definition 3.4. A subset $S$ of a PBA $P$ is called a section of $P$, if it satisfies the following conditions.

(i) $S=[q, p]$ for some $p, q$ such that $q \leqq p$.

(ii) $P=[0, p] \cup[q, 1]$.

$S$ is called proper if $0<q \leqq p<1$. 
Examples: (i) Hosoi [6] proved that the set $\mathscr{L}$ of all intermediate logics is a PBA. Each slice $\mathscr{S}_{n}(n=1,2, \ldots, \omega)$ is a section of $\mathscr{L}$.

(ii) If two PBAs $A$ and $B$ are patched to make a PBA $A \diamond_{f} B$ then $A \cap B$ is a section of $A \diamond_{f} B$.

Now we want to consider the following problem presented by Hosoi and Ono $[8]$ :

"By what operations can all finite PBAs be obtained from 1-cube $S_{1}$ ?",

As Hosoi [4] has remarked, Cartesian product and the pile operation are not sufficient, since the PBA of (Fig. 2) can not be obtained from $S_{1}$ by these operation.

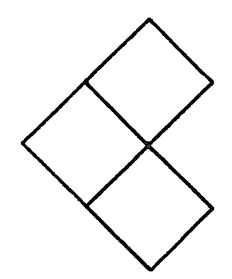

Fig. 2

We shall show that Cartesian product and the patch operation generate all the finite PBAs from $S_{1}$. Further, we shall see that Cartesian product is needed only to obtain $n$-cube $S_{1}^{n}$. To show this we prepare two lemmas.

Lemma 3.5. If $P$ is an $n$-cube, then it has no proper section.

Proof. Let $p_{1}, \ldots, p_{n}$ be the collection of the maximal elements in $P-\{1\}$. Suppose $P$ had a proper section $[q, p]$. Then, since $0<q \leqq p$ $<1$, there exist some $L, K$ such that $\phi \varsubsetneqq L \subset K \varsubsetneqq\{1,2, \ldots, n\}$ and $p=\underset{i \in L}{\wedge} p_{i}$, $q=\wedge_{i \in K} p_{i}$. Take any $j \notin K$. Then $p_{j}$ is neither in $[q, 1]$ nor in $[0, p]$. This is a contradiction.

Lemma 3.6. If a finite $P B A P$ is not a cube, then it has a proper section.

Proof. As an inductive hypothesis, we assume that the theorem holds 
for any PBA whose cardinality is less than the cardinality of $P$. Since $P$ is finite, there exists a maximal element $p$ in $P-\{1\}$. Let $r_{1}, r_{2}, \ldots, r_{k}$ be the enumeration of the elements such that $p \vee r_{j}=1$ Put $r=\wedge_{j=1}^{k} r_{j}$. Then, $p \vee r=p \vee \bigwedge_{j=1}^{k} r_{j}=\bigwedge_{j=1}^{k}\left(p \vee r_{j}\right)=1$. Thus $r$ is the least element such that $p \vee r=1$. Put $q=p \wedge r$.

We shall show that $P=[0, p] \cup[q, 1]$. Suppose $x \notin[0, p]$. Then since $p$ is maximal in $P-\{1\}, p \vee x=1$. Hence $x \geqq r>q$. That is, $x \in$ $[q, 1]$. Now, if $q>0$, then we have a proper section $[q, p]$ in $P$. If $q=0$, then $P \cong[p, 1] \times[r, 1] \cong S_{1} \times[r, 1]$, since $p \vee r=1$ and $p \wedge r=q=$ 0 . Since $P$ is not a cube, $[r, 1]$ is also not a cube. Hence it has a proper section, say, $\left[q^{\prime}, p^{\prime}\right]$ by the inductive hypothesis. Then it is easy to see that $\left[q^{\prime} \wedge p, p^{\prime}\right]$ is a proper section of $P$.

From the above two lemmas we have the following

Theorem 3.7. Any finite $P B A$ can be constructed from $S_{1}$ by Cartesian product and by the patch operation, where Cartesian product is necessary only to obtain $n$-cube $S_{1}^{n}$ from $S_{1}$.

Remark. Let $\boldsymbol{P}$ be the set of all finite PBA and let $\boldsymbol{C}=\left\{S_{1}, S_{1}^{2}, \ldots\right\}$. For any subset $\boldsymbol{S}$ of $\boldsymbol{P}$, we define $\overline{\boldsymbol{S}}$ to be the smallest set of finite PBAs such that $\overline{\boldsymbol{S}} \supset \boldsymbol{S}$ and $\overline{\boldsymbol{S}}$ is closed under the patch operation. Then we can see that $\overline{\boldsymbol{S}}=\boldsymbol{P}$ iff $\boldsymbol{S} \supset \boldsymbol{C}$.

Theorems 2.2 and 3.7 give us a very useful criterion to determine whether a partially ordered set is a PBA or not when it is given in the form of Hasse diagram.

\section{§4. The Height of Models}

In this section, we give a characterization of slice. First we define the notion of normal chains.

Definition 4.1. Let $M$ be a model. A chain in $M$ of length $n$ is a finite sequence $\left(c_{i}\right)_{0 \leqq i \leqq n}$ of elements in $M$ such that $c_{0}<c_{1}<\cdots<c_{n}$. $A$ chain $\left(c_{i}\right)_{0 \leqq i \leqq n}$ is normal if $c_{i} \rightarrow c_{i-1}=c_{i-1}(1 \leqq i \leqq n)$. The length of $a$ 
chain $\alpha=\left(c_{i}\right)_{0 \leqq i \leqq n}$ will be denoted as $l(\alpha)$.

Definition 4.2. Let $M$ be a model. The height $h(M)$ of the model $M$ is $\sup \{l(\alpha) \mid \alpha$ is a normal chain in $M\}$.

Definition 4.3. We define wffs $P_{n}(n=0,1,2, \ldots)$ inductively as follows:

$$
\left\{\begin{array}{l}
P_{0}=a_{0}, \\
P_{i}=\left(\left(a_{i} \rightarrow P_{i-1}\right) \rightarrow a_{i}\right) \rightarrow a_{i} \quad(i \geqq 1) .
\end{array}\right.
$$

Now we state our main theorem in this section.

Theorem 4.4. Let $M$ and $N$ be two models. If $L(M)=L(N)$ then $h(M)=h(N)$.

To prove this we prepare some lemmas.

Lemma 4.5. $S_{n} \supset L(M)$ iff $\quad h(M) \geqq n$.

Proof. Suppose $S_{n} \supset L(M)$. Let $L_{n}=\left\{c_{0}, c_{1}, \ldots, c_{n}\right\}$, where $c_{0}<c_{1}<$ $\cdots<c_{n}$. Clearly $\left(c_{i}\right)_{0 \leqq i \leqq n}$ is a normal chain. Let $f$ be an assignment function such that $f\left(a_{i}\right)=c_{i}$. It is easy to see that $f\left(P_{i}\right)=c_{i}(0 \leqq i \leqq n)$. Hence $f\left(P_{n-1}\right)=c_{n-1}<c_{n} \leqq 1$. Thus $P_{n-1} \notin S_{n}$. This implies $P_{n-1} \notin L(M)$, since $S_{n} \supset L(M)$. Therefore we have an assignment function $g$ into $M$ such that $g\left(P_{n-1}\right)<1$. Let us put $d_{i}=g\left(P_{i}\right) \quad(i \geqq 0)$. Now by Lemma 4.3 in [5], the following (a) and (b) are provable in $L J$.

$$
\begin{array}{ll}
\text { (a) } \quad P_{i-1} \rightarrow P_{i} \equiv a_{0} \rightarrow a_{0} & (i \geqq 1), \\
\text { (b) } \quad P_{i} \rightarrow P_{i-1} \equiv P_{i-1} & (i \geqq 1) .
\end{array}
$$

By (a) we have $d_{i-1} \leqq d_{i}$. Suppose $d_{i-1}=d_{i}$. Then by (b), $d_{i-1}=d_{i} \rightarrow$ $d_{i-1}=1$. Combining these results, since $d_{n-1} \neq 1$, we have $d_{0}<d_{1}<\cdots<$ $d_{n}$. Again by (b), $\left(d_{i}\right)_{0 \leqq i \leqq n}$ is a normal chain of length $n$. Thus $h(M)$ $\geqq n$.

Now we prove the converse. Suppose $h(M) \geqq n$. Then there is a normal chain $\left(c_{i}\right)_{0 \leqq i \leqq n}$ of length $n$. It is easy to see that $C=\left\{c_{0}, c_{1}, \ldots\right.$, $\left.c_{n-1}, 1\right\}$ is a subalgebra of $M$, i.e., closed under the four logical operations, 
It is also clear that $C$ is isomorphic with $L_{n}$. Hence $S_{n} \supset L(M)$.

If we check the proof of the sufficiency of Lemma 4.5 we have the following

Corollary 4.6. $\quad P_{n-1} \notin L(M) \quad$ iff $\quad h(M) \geqq n$.

Now the proof of Theorem 4.4 is immediate from Corollary 4.6. We also obtain the following theorem which characterizes slice.

Theorem 4.7. $L(M) \in \mathscr{S}_{n} \quad$ iff $\quad h(M)=n$.

By our characterization of slice we can prove the following theorem in $[5]$.

Theorem 4.8. If $L(M) \in \mathscr{S}_{m}$ and $L(N) \in \mathscr{S}_{n}$ then $L(M \uparrow N) \in \mathscr{S}_{m+n}$.

Proof. By the hypothesis, we have two normal chains $\left(c_{i}\right)_{0 \leqq i \leqq m}$ in $M$ and $\left(d_{i}\right)_{0 \leqq i \leqq n}$ in $N$. It is easy to see that $c_{0} \rightarrow d_{n-1}=d_{n-1}$. Hence $d_{0}, d_{1}, \ldots, d_{n-1}, c_{0}, c_{1}, \ldots, c_{m}$ is a normal chain of length $m+n$. Hence $h(M \uparrow N) \geqq m+n$. Now suppose $h(M \uparrow N)>m+n$. Then we have a normal chain $\left(c_{i}\right)_{0 \leqq i \leqq m+n+1}$ in $M \uparrow N$. Clearly there is some $k$ such that $c_{k-1} \in N-\left\{1_{N}\right\}$ and $c_{k} \in M$. Then $c_{0}, c_{1}, \ldots, c_{k-1}, 1_{N}$ is a normal chain of length $k$, and $c_{k}, c_{k+1}, \ldots, c_{m+n+1}$ is a normal chain of length $m+n+1-k$. Hence $k \leqq n$, and $m+n+1-k \leqq m$. That is, $n+1 \leqq k \leqq n$. This is a contradiction.

Following Ono [10], a Kripke model is a partially ordered set. Let $K$ be a Kripke model. A subset $J$ of $K$ is called closed if $p \in J$ and $q \geqq$ $p$ implies $q \in J$. It is a well-know fact that the set $P_{K}$ of all closed subsets of $K$ is a model, i.e., a PBA. It can be easily seen that $K$ and $P_{K}$ is characteristic for the same logic (see [1], [2]). We write $L^{*}(K)$ for the logic characterized by $K$. For the definition of the height of $K$ (denoted as $\left.h^{*}(K)\right)$, we refer to $[10]$.

Now using Theorem 4.7, we give another proof for the following theorem due to Ono, 
Theorem 4.9. If $h^{*}(K)=n$ then $L^{*}(K) \in \mathscr{S}_{n}(1 \leqq n \leqq \omega)$.

Proof. It suffices to prove the case that $n$ is finite. Since $h^{*}(K)=n$, we have a chain in $K$ such that

$$
c_{1}<c_{2}<\cdots<c_{n} .
$$

For any $c \in K$, put $T_{c}=\{d \mid d \$ c\}$. Clearly $T_{c} \in P_{K}$. We prove that if $c<c^{\prime}$ then $T_{c} \rightarrow T_{c^{\prime}}=T_{c^{\prime}}$. Put $R=T_{c} \rightarrow T_{c^{\prime}}$. Then

$$
T_{c} \cap R \subset T_{c^{\prime}},
$$

and $R \supset T_{c^{\prime}}$. Suppose $R \supsetneqq T_{c^{\prime}}$ and let $d$ be any element in $R-T_{c^{\prime}}$. Then $d \leqq c^{\prime}$, since $d \notin T_{c^{\prime}}$. This implies $c^{\prime} \in R$, since $d \in R$ and $R$ is closed. On the other hand, $c^{\prime} \in T_{c}$ since $c<c^{\prime}$. Hence by (2), $c^{\prime} \in T_{c^{\prime}}$. This is a contradiction.

Therefore the following chain in $P_{K}$ is a normal chain of length $n$ :

$$
K \supsetneqq T_{c_{1}} \supsetneqq T_{c_{2}} \supsetneqq \cdots \supsetneqq T_{c_{n}} \text {. }
$$

Thus,

$$
h\left(P_{K}\right) \geqq h^{*}(K) .
$$

Now suppose $m=h\left(P_{K}\right)$. Then we have the following normal chain in $P_{K}: N_{0} \varsubsetneqq N_{1} \varsubsetneqq \cdots \varsubsetneqq N_{m}$.

Let $d_{1}$ be any element in $N_{m}-N_{m-1}$. For any $c \in K$, put $M_{c}=\{d \mid d \geqq c\}$. Clearly $M_{c} \in P_{K}$. We have $N_{m-1} \cap M_{d_{1}} \not N_{m-2}$, since otherwise $M_{d_{1}} \subset$ $N_{m-2}$. Let $d_{2}$ be any element in $\left(N_{m-1} \cap M_{d_{1}}\right)-N_{m-2}$. Then $d_{2} \in N_{m-1}$ $-N_{m-2}$ and $d_{2}>d_{1}$. Continuing the same process, we have the following chain in $K: d_{1}<d_{2}<\cdots<d_{m}$. Hence,

$$
h\left(P_{K}\right) \leqq h^{*}(K) .
$$

By (3) and (4), we get $h\left(P_{K}\right)=h^{*}(K)$.

\section{§5. Applications}

Let us consider a finite PBA $P$. Let $a$ be any element in $P$ and let $b_{1}, b_{2}, \ldots, b_{k}$ be the enumeration of elements such that $b_{j} \rightarrow a=a$. (Since $1 \rightarrow a=a, k \geqq 1$.) Put $b=b_{1} \wedge \cdots \wedge b_{k}$. Then $b \rightarrow a=\left(b_{1} \wedge \cdots \wedge b_{k}\right) \rightarrow a=b_{1} \rightarrow$ 
$\left(b_{2} \rightarrow \cdots\left(b_{k-1} \rightarrow\left(b_{k} \rightarrow a\right)\right) \cdots\right)=a$. This means that $b$ is the least element such that $b \rightarrow a=a$. Define a mapping $\lambda: P \rightarrow P$ by $\lambda(a)=b$.

Now we define a sequence $\left\{c_{i}\right\}_{i=0, \ldots}$ as follows:

$$
\left\{\begin{array}{l}
c_{0}=0, \\
c_{k+1}=\lambda\left(c_{k}\right) \quad(k \geqq 0) .
\end{array}\right.
$$

We call this sequence the central sequence in $P$. We can easily see that if $n$ is the least integer such that $c_{n}=1$, then $c_{0}=c_{n+1}=0$ and $c_{0}<c_{1}<$ $\cdots<c_{n}=1$. This sequence has the following property.

Theorem 5.1. Let $\left\{c_{i}\right\}$ be the central sequence in $P$, and $n$ be the least integer such that $c_{n}=1$. Then $h(P)=n$.

Proof. Since $0=c_{0}<c_{1}<\cdots<c_{n}=1$ is a normal chain, $h(P) \geqq n$. Suppose $h(P)>n$. Then we have a normal chain $d_{0}<d_{1}<\cdots<d_{n}<d_{n+1}$. Clearly $c_{0} \leqq d_{0}$. Then from the next Lemma 5.2, $d_{1} \rightarrow c_{0}=c_{0}$. Hence $c_{1} \leqq d_{1}$. Continuing the same process, we see that $c_{2} \leqq d_{2}, \ldots, c_{n} \leqq d_{n}$. Thus we obtain $1=c_{n} \leqq d_{n}<d_{n+1}$. This is a contradiction.

Lemma 5.2. If $a>b \geqq c$ and $a \rightarrow b=b$, then $a \rightarrow c=c$.

Proof. Let $d=a \rightarrow c$. Then $a \wedge d=a \wedge(a \rightarrow c) \leqq c \leqq b$. On the other hand, $a \rightarrow b=b$. Hence $d \leqq b$. Since $b<a, d=a \wedge d=a \wedge(a \rightarrow c) \leqq c$. Clearly $d=a \rightarrow c \geqq c$. Therefore $d=c$.

Theorem 5.3. If $h(P)=n$, then $\left[c_{i}, c_{i+1}\right]$ is a cube.

Proof. Since other cases can be proved similarly, we only show that $\left[0, c_{1}\right]$ is a cube. Let $\left\{p_{1}, p_{2}, \ldots, p_{k}\right\}$ be the set of minimal elements in $P-\{0\}$. Then it is easy to see that $x \rightarrow 0=0$ iff $x \geqq p_{j}$ for $1 \leqq j \leqq k$. Hence $c_{1}=\bigvee_{j=1}^{k} p_{j}$. Then from Corollary 4.3 we have that $\left[0, c_{1}\right]$ is a cube.

By Theorem 5.1 and 5.3 we can easily calculate the height of a model if its Hasse diagram is given.

A logic $L$ is called an immediate predecessor of another logic $L^{\prime}$ if $L \varsubsetneqq L^{\prime}$ and there are no logics between $L$ and $L^{\prime}$. We write $L<L^{\prime}$ to 
denote that $L$ is an immediate predecessor of $L^{\prime}$.

Concerning immediate predecessors of $S_{n}$, Hosoi proved that if $n \geqq 3$ then $S_{n+1}<S_{n}, S_{n} \cap S_{1} \uparrow S_{1}^{2}<S_{n}$, and $S_{n} \cap S_{1} \uparrow S_{1}^{2} \uparrow S_{1}<S_{n}$. (See Ono [11].) Since $\mathscr{L}$ is a PBA, we can apply Theorem 3.2 to obtain the following theorem. We owe this remark to Prof. T. Hosoi.

Theorem 5.4. $\quad S_{n} \cap S_{1} \uparrow S_{1}^{2} \cap S_{1} \uparrow S_{1}^{2} \uparrow S_{1}<S_{n} \cap S_{1} \uparrow S_{1}^{2}$,

$$
\begin{aligned}
& S_{n} \cap S_{1} \uparrow S_{1}^{2} \cap S_{1} \uparrow S_{1}^{2} \uparrow S_{1}<S_{n} \cap S_{1} \uparrow S_{1}^{2} \uparrow S_{1}, \\
& S_{n+1} \cap S_{1} \uparrow S_{1}^{2}<S_{n} \cap S_{1} \uparrow S_{1}^{2}, \quad \text { and } \\
& S_{n+1} \cap S_{1} \uparrow S_{1}^{2} \uparrow S_{1}<S_{n} \cap S_{1} \uparrow S_{1}^{2} \uparrow S_{1} .
\end{aligned}
$$

\section{References}

[1] Birkhoff, G., Lattice theory, American Mathematical Society Colloquium Publications 25, 1948.

[2] de Jongh, D.H.J. and A.S. Troelstra, On the connection of partially ordered sets with some pseudo-Boolean algebras, Indag. Math., 28 (1966), 317-329.

[3] Dummett, M., A propositional caluculus with denumerable matrix, J. Symbolic Logic, 24 (1959), 96-107.

[4] Hosoi, T., On the axiomatic method and the algebraic method for dealing with propositional logics, J. Fac. Sci., Univ. Tokyo. Sec. I, 14 (1967), 131-169.

[5] - On intermediate logics I, Ibid., 14 (1967), 293-312.

[6] - - , On intermediate logics II, Ibid., 16 (1969), 1-12.

[7] Hosoi, T. and H. Ono, Axiomatization of models for intermediate logics constructed with Boolean models by piling up, Publ. RIMS, Kyoto Univ., 8 (1972), 111.

[8] - Intermediate propositional logics (A survey), J. of Tsuda college, 5 (1973), 67-82.

[9] McKay, C.G., On finite logics, Indag. Math., 29 (1967), 363-365.

[10] Ono, H., Kripke models and intermediate logics, Publ. RIMS, Kyoto Univ., 6 (1970), 461-476.

[11] - Some results on the intermediate logics, Ibid., 8 (1972), 117-130.

[12] Rasiowa, H. and R. Sikorski, The mathematics of meta-mathematics, Monografie Mathematyczne 41, Warszawa, 1963. 
